\title{
Rückblick Jahrestagung 2014 der DGRPÄC, VDÄPC und ÖGPÄRC in München
}

\author{
Retrospect of Conjoined German and Austrian Societies of Plastic Surgery Annual Meeting 2014 in Munich
}

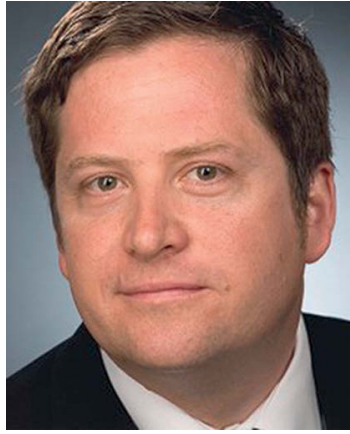

Univ.-Prof. Dr. med. Riccardo E. Giunta

Bibliografie

DOI http://dx.doi.org/ $10.1055 / \mathrm{s}-0034-1395684$ Handchir Mikrochir Plast Chir 2014; 46: 323-324 (c) Georg Thieme Verlag KG Stuttgart · New York ISSN 0722-1819

Korrespondenzadresse Univ.-Prof. Dr. med. Riccardo E. Giunta Handchirurgie, Plastische Chirurgie und Ästhetische Chirurgie

Klinikum der Ludwig-

Maximilians Universität (LMU) München Pettenkoferstraße 8a 80336 München

r.giunta@med.uni-muenchen.de
Im September fand die gemeinsame Jahrestagung der Deutschen Gesellschaft der Plastischen, Rekonstruktiven und Ästhetischen Chirurgen (DGPRÄC), der Vereinigung der Deutschen Ästhetisch-Plastischen Chirurgen (VDÄPC) und der Österreichischen Gesellschaft für Plastische, Ästhetische und Rekonstruktive Chirurgie (ÖGPÄRC) bei uns in München statt. Gemeinsam mit Lars Kamolz aus Graz und Günther Machens vom Klinikum rechts der Isar hatten wir 3 als Kongresspräsidenten die Gelegenheit, die Jahrestagung auszugestalten. Unser Ziel als akademische Plastische Chirurgen war es eine Tagung zu organisieren, die einerseits die Plastische Chirurgie mit Ihren 4 Säulen Rekonstruktive Chirurgie, Handchirurgie, Ästhetische Chirurgie und Verbrennungschirurgie mit dem Fundament Forschung verbindet und andererseits die Jahrestagung interdisziplinär und international zu öffnen.

Umgesetzt haben wir dieses Konzept zunächst mit insgesamt 4 Kursen mit großen Themen der beiden Münchner Universitätskliniken vor Beginn des eigentlichen Kongresses: So fanden 2 OP Kurse zu den Themen „Handgelenksarthroskopie“ und „Mikro-Lymphgefäßchirurgie“ und 2 Präparationskurse zu den Themen „Freie Lappenplastiken“ und „Facelifting“ statt. Jeweils eine hochkarätige internationale Faculty machte die Kurse sehr attraktiv. Besonders die an der Anatomischen Anstalt der Ludwig-Maximilians Universität durchgeführten beiden Präparationskurse an Frischpräparaten fanden sehr guten Zuspruch, sodass wir diese gerne in regelmäßigen Abständen unabhängig vom Kongress wiederholen werden. Der internationalen Faculty und der Anatomischen Anstalt der LMU sowie allen Anderen, die zum Erfolg dieser Kurse beigetragen haben, gebührt großer Dank.

Im Rahmen dieser Kurse konnten auch die Synergieeffekte als Kongresspräsident und Herausgeber dieser Zeitschrift durch einen Beitrag von Giovanni Botti [1] zum Thema des ästhetischen Präparationskursen nutzen: Dieser steht im Open Access kostenlos zur Verfügung und bietet über die neue Möglichkeit des QR Codes die an sich vorgegebene Zweidimensionalität einer Zeitschrift zu durchbrechen und um das Medium Video zu erweitern.

Liebe Leser,

testen Sie diese neue Möglichkeit, die gerade im Hinblick auf OP Techniken, funktionelle Ergebnisse an der oberen Extremität oder auch für arthroskopische oder z.B. sonografisch bewegte Bilder aus meiner Sicht besonders attraktiv ist. Darüber hinaus war auch der neue HaMiPla Best Paper Award, der in einer eigenen Sitzung vergeben wurde, eine attraktive Neuerung (siehe „In eigener Sache“) [2].
Die Ausrichtung der Jahrestagung gemeinsam mit der österreichischen Gesellschaft war eine bereits bewährte, große Bereicherung und hat die Tagung internationalisiert. Eine persönliche Note haben die „Transalp Sitzungen“ ( $\bullet$ Abb. 1) mit deutscher, österreichischer und zusätzlich italienischer Beteiligung der Tagung gegeben. Insgesamt 12 italienische Kollegen haben das Programm bereichert und gemeinsam mit den anderen Kollegen aus Europa einen europäischen Charakter gegeben. Die Fördermittel der Deutschen Forschungsgemeinschaft (DFG) haben dies ermöglicht, der an dieser Stelle ebenfalls Dank gebührt. Weiterhin konnte noch das Chinesisch-Deutsches Symposium zum Thema „Reconstruction of Complex Defects after Trauma and Tumor Sino-German Network Meeting on Advances in Basic Science and Clinical Strategies“ in die Jahrestagung inkludiert werden. Das ChinesischDeutsche Zentrum für Wissenschaftsförderung (CGS) hat dankenswerterweise die Fördermittel für diese wichtige Veranstaltung bereit gestellt und damit ermöglicht, dass 15 Kollegen aus ganz China Gelegenheit zu einem sehr fruchtbaren Wissensaustausch und zum Aufbau von künftigen Kooperationen nach Deutschland reisen konnten ( 0 Abb. 2). Meinen beiden Mitarbeitern Frau Tanja Herrler, die derzeit in der Plastischen Chirurgie am $9^{\text {th }}$ People Hospital bei Prof. Li in Shanghai tätig ist, und unserem Stipendiaten seit 2012 aus Wuhan Lin Shenyu sei für die umfangreichen Vorarbeiten gedankt.

Mein persönliches Anliegen war es auch berufspolitischen Themen ein größeres Forum zu geben. Insofern möchte ich bei gerade bei den hochkarätigen Teilnehmern ( $\boldsymbol{O}$ Abb. $\mathbf{3}$ ) unseres Fachgebiets, anderer chirurgischer Fachgebiete und aus dem Bayerischen Ministerium für Wissenschaft, Forschung und Kunst für ihre Beiträge an der Podiumsdiskussion zur „Stellung der Plastischen Chirurgie an Kliniken" herzlich bedanken. Nach wie vor bilden leider nur zu wenige Kliniken die moderne Organisationsstruktur der Chirurgie mit der Plastischen Chirurgie als eigenständiges, gleichgestelltes Fachgebiet in der großen Familie der chirurgischen Fachgebiete ab.

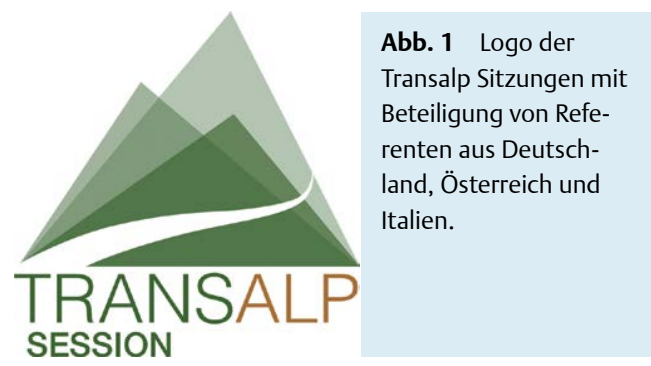




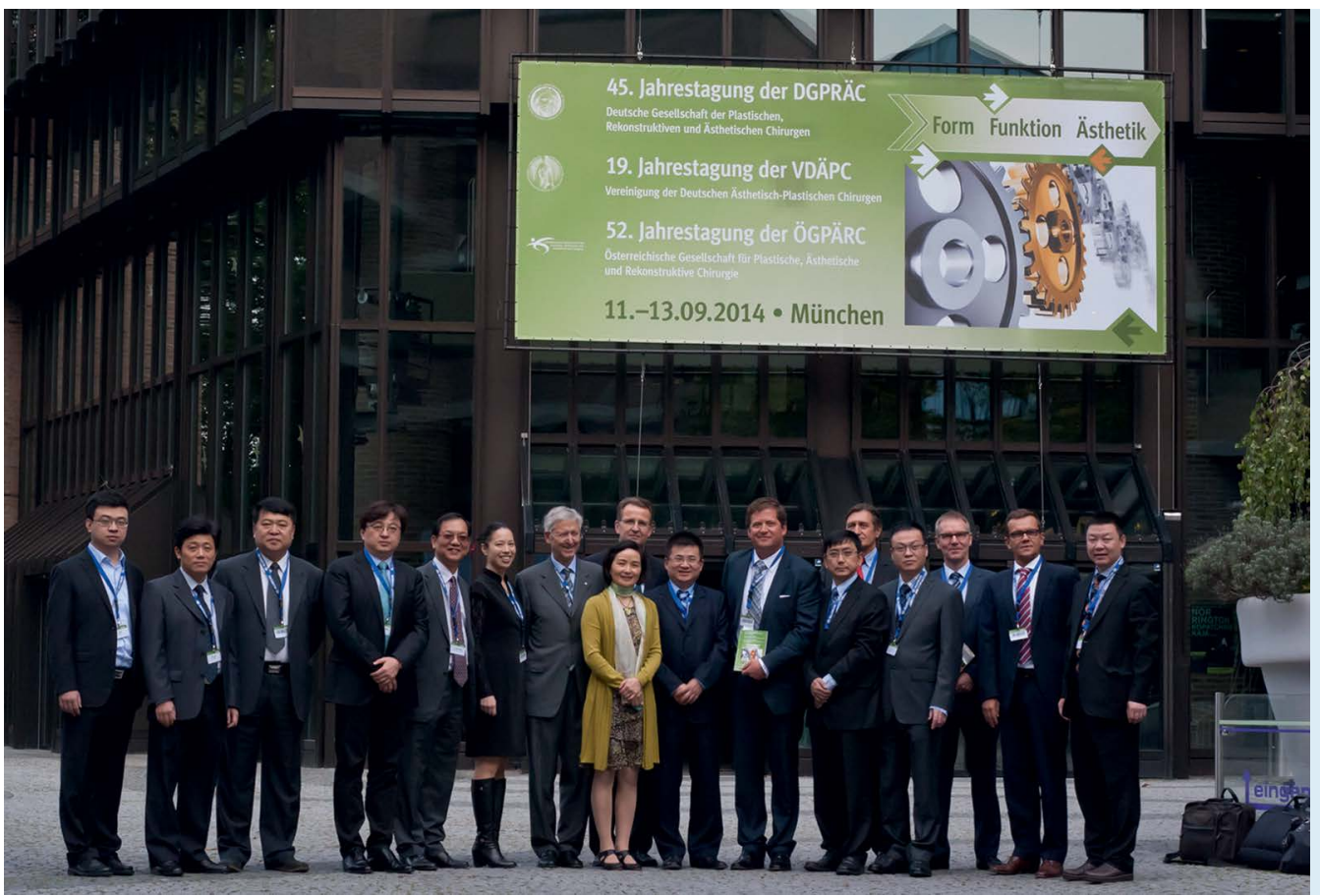

Abb. 2 Die Teilnehmer der Eröffnungsveranstaltung des Chinesisch-Deutschen Symposiums „Reconstruction of Complex Defects after Trauma and Tumor - Sino-German Network Meeting on Advances in Basic Science and Clinical Strategies“.

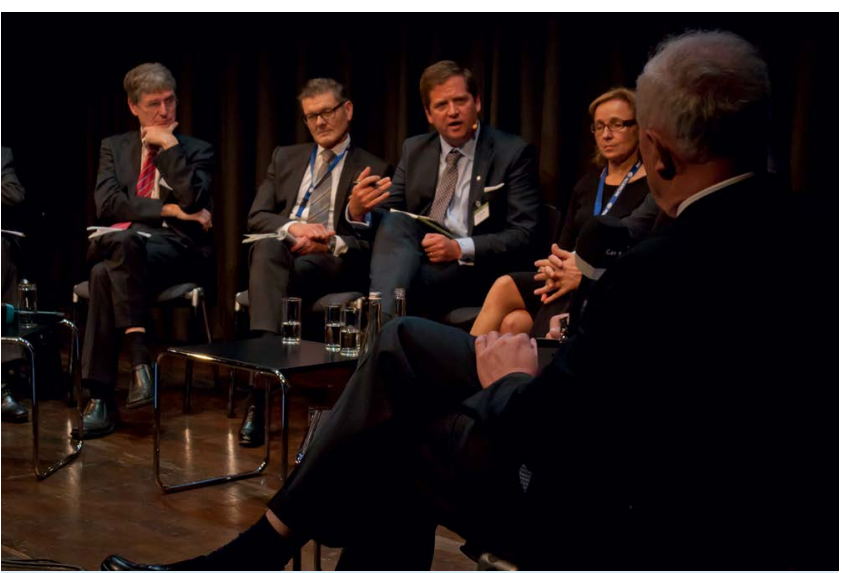

Abb. 3 Podiumsdiskussion zur Stellung der Plastischen Chirurgie an Kliniken mit (von links nach rechts) Ministerialdirigent Ulrich Hörlein, Peter Vogt (Präsident der Deutschen Gesellschaft für Chirurgie), Riccardo Giunta (Moderator), Jutta Liebau (Präsidentin der DGPRÄC), seitlich: Norbert Haas (Charité Berlin), nicht abgebildet: Hartwig Bauer (ehemaliger Genralsekretär der Deutschen Gesellschaft für Chirurgie, Hans-Günther Machens (München), Lukas Prantl (Regensburg), Lars-Peter Kamolz (Graz).

Veranstaltungen dieser Art tragen zum wechselseitigen Verständnis und Anerkennung bei und unterstützen die Modernisierung der Organisationsstrukturen an Kliniken mit dem Ziel einer insgesamt modernen und damit starken Gesamtchirurgie. Der Samstag hatte ganztags zusätzlich Patienteninformationsveranstaltungen ebenfalls zu allen 4 Säulen der Plastischen Chirurgie ( $\bullet$ Abb. 4). Der Gasteig als Kongressort mit seinem regen Publikumsverkehr durch Stadtbibliothek und Volkshochschule bot sich hierfür an. Aus meiner Sicht ist es besonders wichtig auch einer breiten Öffentlichkeit zu vermitteln, dass Plastische Chirurgie nicht wie meist angenommen, mit „Schönheitschirurgie“" gleichzusetzen ist. Dies ist umso bedeutender als unter diesem suggerierten Bild des Plastischen Chirurgen oft auch das Ansehen des Plastischen Chirurgen in der Peer Community leidet $[3,4]$. Insgesamt wäre die Arbeit an einer verbesserten Wahrnehmung des Fachgebiets meines Erachtens einer

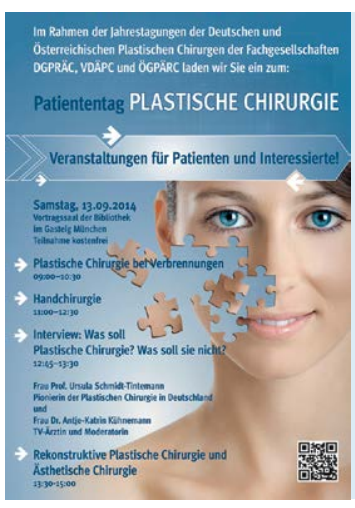

Abb. 4 Plakat zum Patiententag.

der wichtigsten Schwerpunkte für die künftige Öffentlichkeitsarbeit der Gesellschaft.

Insgesamt hat die Tagung mit 49 Sitzungen, 435 Vorträgen, 137 wissenschaftlichen Poster, 9 Lunchsymposien, 5 Workshops sowie den erwähnten Präparations- und Operationskursen regen Zuspruch gefunden. Mit insgesamt fast 1400 registrierten Teilnehmern war dies eine der best besuchten Jahrestagungen. Dies wiederum dokumentiert das rege, stetig steigende Interesse an unserem Fachgebiet Plastische Chirurgie. Allen Beteiligten, der Kongressfirma, den vielen Mitwirkenden und verschiedensten Unterstützern der Veranstaltung sei an dieser Stelle nochmals ganz herzlich gedankt!

München im November 2014 Riccardo Giunta

\section{Literatur}

1 Botti G, Botti C. Midface Lifting - Our current Approaches. Handchir Mikrochir Plast Chir 2014; 46: 224-233

2 Maier K. HaMiPla Best Paper Sitzung bei der DGPRÄC: Jahrestagung in München - Ihre Favoriten 2013. Handchir Mikrochir Plast Chir 2014; 46: 383-384

3 Giunta RE. Gedankenaustausch zum 90sten Geburtstag von Frau Prof. Ursula Schmidt-Tintemann: Was soll Plastische Chirurgie? Was soll sie nicht? Handchir Mikrochir Plast Chir 2014; 46: 203-205

4 Giunta RE, Schmidt-Tintemann U. Was soll Plastische Chirurgie? Und was soll sie nicht? Handchir Mikrochir Plast Chir 2013; 45: 191-192 